\section{Latent pregnancy-associated hypopituitarism accompanied by chronic thyroiditis and limited scleroderma, revealed by the breakout of adrenal insufficiency $\mathbf{4 2}$ years after delivery}

\author{
Shuzo Kaneko, 1 Manabu Shiozaki,1 \\ Nariko Omori,2 Shinji Tsuruta, \\ Hitomi Haraoka, ${ }^{1}$ Yusuke Tsukamoto' 1 \\ 1Department of Internal Medicine, \\ Itabashi Chuo Medical Center, Tokyo; \\ 2Department of Internal Medicine, IMS \\ Memorial Hospital, Tokyo, Japan
}

\section{Abstract}

Pregnancy-associated hypopituitarism (PAH) has been referred to as Sheehan's syndrome and lymphocytic hypophysitis, both of which are occasionally disclosed several decades after delivery. We report the rare case of a 70year-old woman diagnosed with $\mathrm{PAH}$ with empty sella 42 years after her second delivery; she had had an episode of hypovolemic shock during her second delivery. She had been referred to our hospital because of the emergence of non-sustained ventricular tachycardia and symptoms of appetite loss and general fatigue accompanied by hyponatremia, hypoglycemia, and hypothyroidism. Hypopi-tuitalism and secondary adrenal insufficiency had been revealed after her hospitalization. She had suffered from Raynaud's syndrome for the past several decades. Our testing revealed that the patient had autoantibodies for thyroid peroxidase, thyroglobulin, and centromere, indicating the diagnosis of chronic thyroiditis and limited scleroderma. Her series of symptoms had been treated successfully by hydrocortisone and thyroid hormone replacement therapy. To the best of our knowledge, latent PAH accompanied by limited scleroderma have not been described in the international literature to date though quite a few latent cases may exist. We assume that some autoimmune mechanism during the perinatal period may possibly be a common step in the pathogenesis of PAH, chronic thyroiditis and limited scleroderma.

\section{Introduction}

Pregnancy-associated hypopituitarism (PAH) has also been referred to as Sheehan's syndrome and lymphocytic hypophysitis.
Sheehan's syndrome classically refers to PAH due to pituitary necrosis occurring during severe hypotension or shock secondary to massive bleeding in the postpartum period, ${ }^{1}$ but in recent years, immunological pathogenesis is also assumed in the disease. ${ }^{2-4}$ Lymphocytic hypophysitis commonly occurs during late pregnancy or in the puerperium, and is thought to be an autoimmune disease, possibly involving both humoral and cell-mediated mechanisms. ${ }^{4,5}$ The diagnosis of several reported cases was delayed for several decades after the pregnancy in both Sheehan's syndrome and lymphocytic hypophysitis, which are revealed by the symptoms of hormonal deficiencies of single or multiple anterior pituitary hormones, or by a detection of empty sella. We herein describe a rare case of latent PAH accompanied by chronic thyroiditis and limited scleroderma, which was revealed by the breakout of adrenal insufficiency 42 years after the delivery.

\section{Case Report}

A 70-year-old woman was referred to our hospital because of the emergence of non-sustained ventricular tachycardia. Four days before her referral, she had been admitted to another hospital because of symptoms of appetite loss and general fatigue. Hyponatremia, hypoglycemia, and hypothyroidism were identified during that hospitalization. She had no medical history of note except for three deliveries at the ages of 25,28 and 30 . During her second delivery, she had an episode of loss of consciousness because of hypovolemic shock secondary to bleeding, and within several years after the last delivery she became aware that her axillary and pubic hair were becoming very sparse.

At our hospital, her physical and laboratory examination results were as follows. Her blood pressure was $112 / 64 \mathrm{mmHg}$, pulse rate $64 / \mathrm{min}$, $\mathrm{SpO}_{2} 96 \%$ (room air), body temperature $36.3^{\circ} \mathrm{C}$. Her height was $155 \mathrm{~cm}$, and weight 52 $\mathrm{kg}$. No goiter was observed. No abnormalities were observed the chest or abdomen. Slight edema was observed in her legs, and sclerodactyly was observed in her fingers. We found that the patient had suffered from Raynaud's syndrome for the past several decades.

On blood examination, the levels of serum total protein and albumin were low at $55 \mathrm{~g} / \mathrm{L}$ (normal: 65-82) and $27 \mathrm{~g} / \mathrm{L}$ (normal: 38-53), respectively. Hyponatremia, hypoglycemia and hypouricemia were observed at $118 \mathrm{mmol} / \mathrm{L}$ (normal: 136-147), $3.3 \mathrm{mmol} / \mathrm{L}$ (normal: 3.9 6.1) and 148.7 umol/L (normal: 154.7-416.4), respectively. The level of creatine kinase (CK) was slightly elevated, but those of CK-MB and troponin I were not. Brain natriuretic peptide
Correspondence: Shuzo Kaneko, Department of Internal Medicine, Itabashi Chuo Medical Center, 2-12-7 Azusawa, Itabashiku, Tokyo, 174-0051, Japan.

Tel. +81.339 .670572 - Fax: +81.339 .670572$

E-mail: shuzo-kaneko10@ob.md.tsukuba.ac.jp

Key words: pregnancy-associated hypopituitarism, empty sella, adrenal insufficiency, chronic thyroiditis, limited scleroderma.

Conflict of interests: the authors report no conflicts of interests.

Contributions: SK, medical management during patient's hospitalization, manuscript writing, data collecting and analyzing, and references search; MS, medical management during patient's hospitalization, data collecting and analyzing, and references search; NO, data collecting and analyzing; ST, HH, YT, medical management during patient's hospitalization

Received for publication: 26 January 2013 Accepted for publication: 22 February 2013.

This work is licensed under a Creative Commons Attribution NonCommercial 3.0 License (CC BYNC 3.0)

(C) Copyright S. Kaneko et al., 2013

Licensee PAGEPress, Italy

Endocrinology Studies 2013; 3:e5

doi:10.4081/es.2013.e5

(BNP) was high, at $206.000 \mathrm{ug} / \mathrm{L}$ (normal:0 20000). Urinalysis showed no proteinuria and no microscopic hematuria. A spot urinary concentration of electrolytes and urinary osmotic pressure (U-osm) showed relative high tonicity and osmolarity [i.e., $\mathrm{U}-(\mathrm{Na}+\mathrm{K})=127+20.3$ $\mathrm{mmol} / \mathrm{L}, \mathrm{U}-\mathrm{osm} 468 \mathrm{mosm} / \mathrm{L}$ ] despite the hyponatremia and low plasma-osmotic pressure (P-osm). On serological examination, IgG, anti-thyroid peroxidase antibody (antiTPO-Ab) and anti-thyroglobulin antibody (antiTG-Ab) were detected at the slightly high levels of $17.87 \mathrm{~g} / \mathrm{L}$ (normal:8.70-17.0), $75.2 \mathrm{U} / \mathrm{mL}$ (normal: 0-50) and 56.5 U/mL (normal: 0-32.7), respectively. IgG subclass 4 was not elevated at $0.09 \mathrm{~g} / \mathrm{L}$ (normal: 0.048-1.05). Antinuclear antigen (ANA) was positive at 1:1280 dilution with a discrete speckled pattern, and anti-centromere-antibody was detected, whereas antibodies for Scl-70 and RNP were not. On endocrine examination, the levels of free-T3 and T4 were low at $2.43 \mathrm{pmol} / \mathrm{L}$ (normal: 3.27 6.25) and $0.10 \mathrm{pmol} / \mathrm{L}$ (normal: 0.11-0.22), respectively. The levels of cortisol (F) and ACTH were low at $63.4 \mathrm{nmol} / \mathrm{L}$ (normal: 104.9 507.8) and $9600 \mathrm{~g} / \mathrm{L}$ (normal: 7200-63300), respectively, even at an early-morning measurement. The level of TSH was slightly high at $7.21 \mathrm{mIU} / \mathrm{L}$ (normal: 0.39-4.01), whereas those of $\mathrm{LH}, \mathrm{FSH}$, and GH were very low at 4170 
mIU/L (normal: <157.800, standard maximal value rise in postmenopausal periods), 6800 mIU/L (normal: $<87.400$, standard maximal value rise in postmenopausal periods), and $0.08 \mathrm{ug} / \mathrm{L}$ (normal: 0.13-9.88), respectively. $\mathrm{ADH}$ was inappropriately detected at $1.7 \mathrm{pg} / \mathrm{mL}$ within normal range $(0.3-4.2 \mathrm{pg} / \mathrm{mL})$ although the patient showed low P-osm and no sign of volume depletion, suggesting a syndrome of inappropriate secretion of ADH (SIADH)-like pattern. A pituitary MRI showed empty sella suggesting hypopituitarism, but anti-pituitary gland antibody was not detected in her serum. A pituitary MRI image was shown in Figure 1.

Just after her admission, the patient's electrocardiogram showed inverted T waves in V26 , and her chest X-ray showed slight cardiomegaly. Echocardiography showed moderate left ventricular hypokinesis in the apex and lateral to the posterior wall, and normal contraction at the base. Three days after admission, the patient's myocardiac contraction improved dramatically without vasoactive agonists, and the T-wave inversion in her electrocardiogram also alleviated significantly, suggesting the clinical course of stress-induced takotsubo cardiomyopathy although coronary angiography was not performed. We suspected that her cardiomyopathy had been induced under the adrenal insufficiency condition secondary to hypopituitarism.

To treat what was essentially a series of symptoms, we started hydrocortisone and thyroid hormone replacement therapy. After the initiation of the replacement therapy, the patient's complaint of general fatigue and appetite loss gradually improved, and her refractory hyponatremia and hypoglycemia showed dramatic improvement. Her clinical course is summarized in Figure 2. During her 43-day hospitalization in our facility, ventricular tachycardia was never detected.

At hospitalization, refractory hyponatremia and hypoglycemia were observed despite an infusion of hypertonic saline and glucose and water restriction. After hydrocortisone (30 $\mathrm{mg} /$ day) and thyroid hormone replacement therapy from day 6 , both the concentration of serum sodium and fasting glucose rose steadily and reached normal ranges, and patient's complaint of general fatigue was dramatically disapeared. As the patient's stable condition continued, the dose of hydrocortisone was tapered to $20 \mathrm{mg}$ per day and she was discharged at day 43 .

During a later period of stable condition, we investigated the standby capacity of the patient's pituitary gland by hormone stimulation test. To stimulate hormone-producing cells in the pituitary anterior lobe, including GH-, TSH-, PRL-, ACTH-, LH-, and FSH-producing cells, we administered a simultaneous injection of growth hormone release peptide 2 (GH-RP-2), thyrotropin-releasing hormone
(TRH), corticotropin-releasing hormone (CRH), and LH-releasing hormone (LH-RH). Low to no response was observed for $\mathrm{GH}$, PRL, ACTH, LH and FSH, whereas TSH showed a near-normal response. We also performed a rapid ACTH stimulation test to evaluate the standby capacity of the patient's adrenal gland. A low cortisol (F) response was observed. We suspected that weak ACTH stimulation over a long period of time might retract the adrenal glands. These results are shown in Figure 3.

In the rapid ACTH test, the F secretion was low at the points of 30 and 60 minutes. The GH-RP-2 test showed low to no response of GH secretion at all time points. The TRH test results showed a near to normal response of TSH secretion, and a low response of PRL secretion. The CRH test showed low to no response of both ACTH and F. The LH-RH test also showed low to no response of both $\mathrm{LH}$ and FSH. Grey zones shows the reference ranges of target hormone in each stimulating tests. (In LH-RH test, reference ranges is shown only for $\mathrm{LH}$, which is relatively sensitive and narrow range compared to $\mathrm{FSH}$.)

\section{Discussion}

PAH has been described as Sheehan's syndrome and lymphocytic hypophysitis. Classically, Sheehan's syndrome is due to pituitary ischemia leading to necrosis during severe hypotension secondary to massive bleeding at the delivery, ${ }^{1}$ and lymphocytic hypophysitis is an autoimmune disease that often occurs during late pregnancy or in the puerperium, and is accompanied by other autoimmune diseases (e.g. chronic thyroiditis) or the detection of anti-pituitary gland anti-

\section{body (APA). ${ }^{4,5}$}

PAH is occasionally identified a long time after a pregnancy, accompanied by empty sella. If an episode of hypovolemic shock at the past delivery is not confirmed and hypopituitarism shows slow clinical progression, it would be difficult to make a definitive diagnosis of PAH. In the present case, hypopituitarism was revealed 42 years after the patient's second delivery by the breakout of adrenal insufficiency, but the patient had been aware of lessening axillary and pubic hair for the past several decades, suggesting that latent hypopituitarism had been slowly developing.

We researched a total of 48 Japanese case reports of hypopituitarism with empty sella that had been described in detail; the patients' average age was $58.5 \pm 14.6$, and there were twice as many females as males (32:16). Most of the female patients had a prior pregnancy

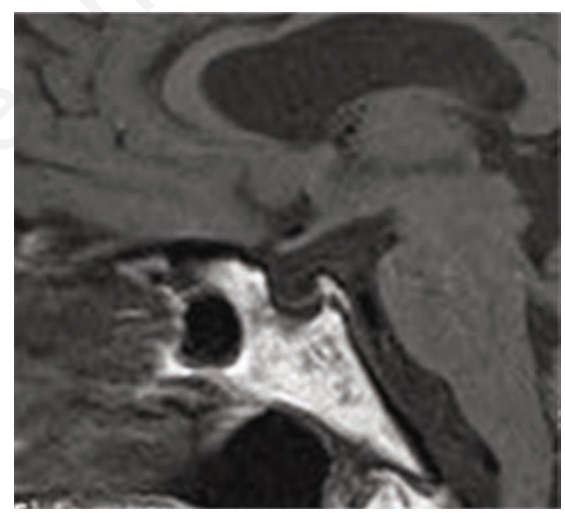

Figure 1. Pituitary magnetic resonance imaging [fluid-attenuated inversion recovery (FLAIR) mode, sagittal section] showing empty sella.

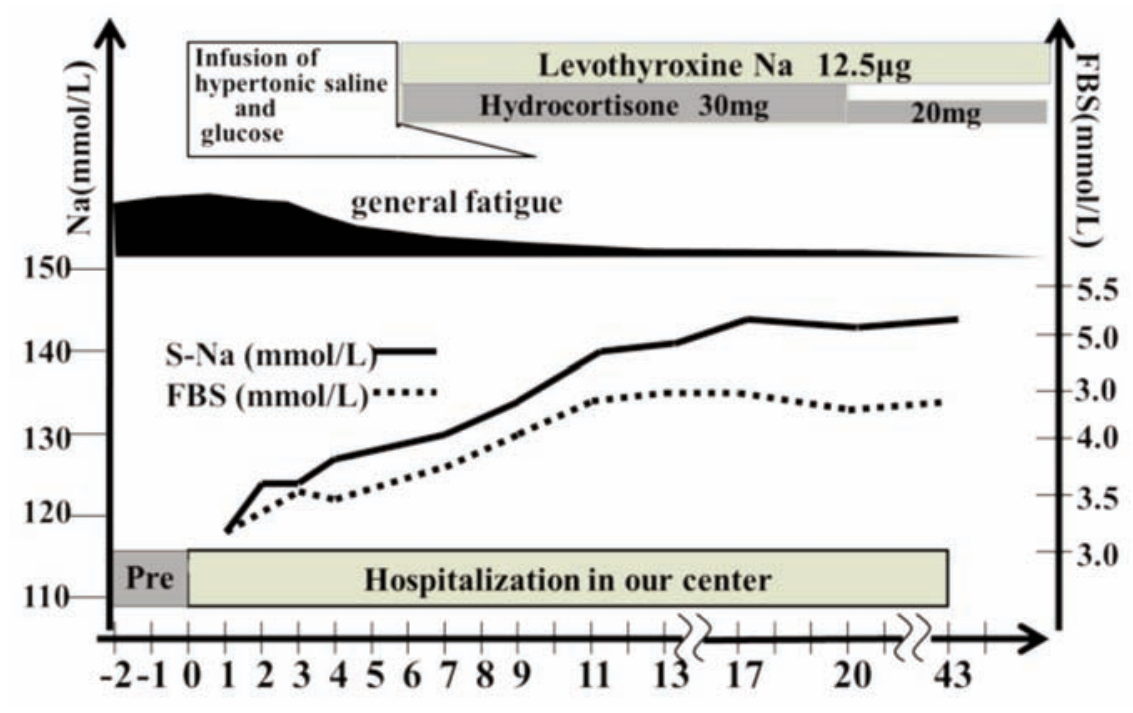

Figure 2. The patient's clinical course. 
(29/32). The origin of the disease was Sheehan's syndrome in 12 cases, an autoimmune disorder in three, and prolactinoma in one case; in 16 cases, the origin was unknown. These data suggest that more PAH patients might be included among the reported cases of hypopituitarism with empty sella, and we suspect that latent or subclinical cases of PAH may also exist.

In recent years, Sheehan's syndrome has been recognized to develop by some type of immunological pathogenesis, because hypopituitarism shows slow clinical progression in some cases, and APA is detected frequently in patients with Sheehan's syndrome. ${ }^{2-4}$ Goswami et al. reported that 12 of 19 (63.1\%) patients with Sheehan's syndrome had APA against the 49-kDa autoantigen (neuron-specific enolase), whereas only four of the 28 (14.2\%) controls without prior pregnancy and five of the 28 (17.8\%) controls with prior pregnancy showed the same result. ${ }^{2}$ De Bellis and co-workers reported that APA (investigated by an indirect immunofluorescence method on cryostat sections of young baboon pituitary gland) were found in seven of 20 (35\%) and anti-hypothalamus antibodies (against non-vasopressinsecreting cells) were found in eight of 20 (40\%) patients with Sheehan's syndrome, whereas none of the controls showed these findings. ${ }^{3}$ The autoimmune mechanism underlying $\mathrm{PAH}$ is unknown, but two mechanisms have been assumed; one is the exposure of pituitary autoantigen to the systemic immune system due to pituitary hyperfusion and enlargement, or necrosis during late pregnancy or the post-partum period. The other is the exposure of autoantigen originated from the placenta which is similar to antigenicity to some proteins from the pituitary gland. The significance of APA detection and its pathogenesis have been controversial, but a recent animal prospective investigation demonstrated serial morphological changes of the pituitary gland from the early phase (showing pituitary swelling) to the late phase (showing empty sella) in mice immunized with pituitary proteins, suggesting a direct immunological pathogenesis. ${ }^{6}$

Our patient had an episode of hypovolemic shock during her second delivery, leading to the diagnosis of Sheehan's syndrome, while pituitary dysfunction seemed to slow the syndrome's progression. We believe that autoimmune mechanisms might have been a step in the development of our patient's hypopituitarism, even though APA was not detected in her serum. Serum APA is detected mainly by using the immunoreaction against an extract from animal/human pituitary gland (including the fraction of cell membrane or cytoplasm), by immunoblotting or indirect immunofluorescence on cryostat sections. ${ }^{3}$ Unfortunately, a reliable method of APA detection is not available at the present time, because both the sensitivity and specificity of the detection of APA for PAH are low, and the results of the various available methods differ markedly.

We evaluated our patient's serum using an indirect immunofluorescence assay against an
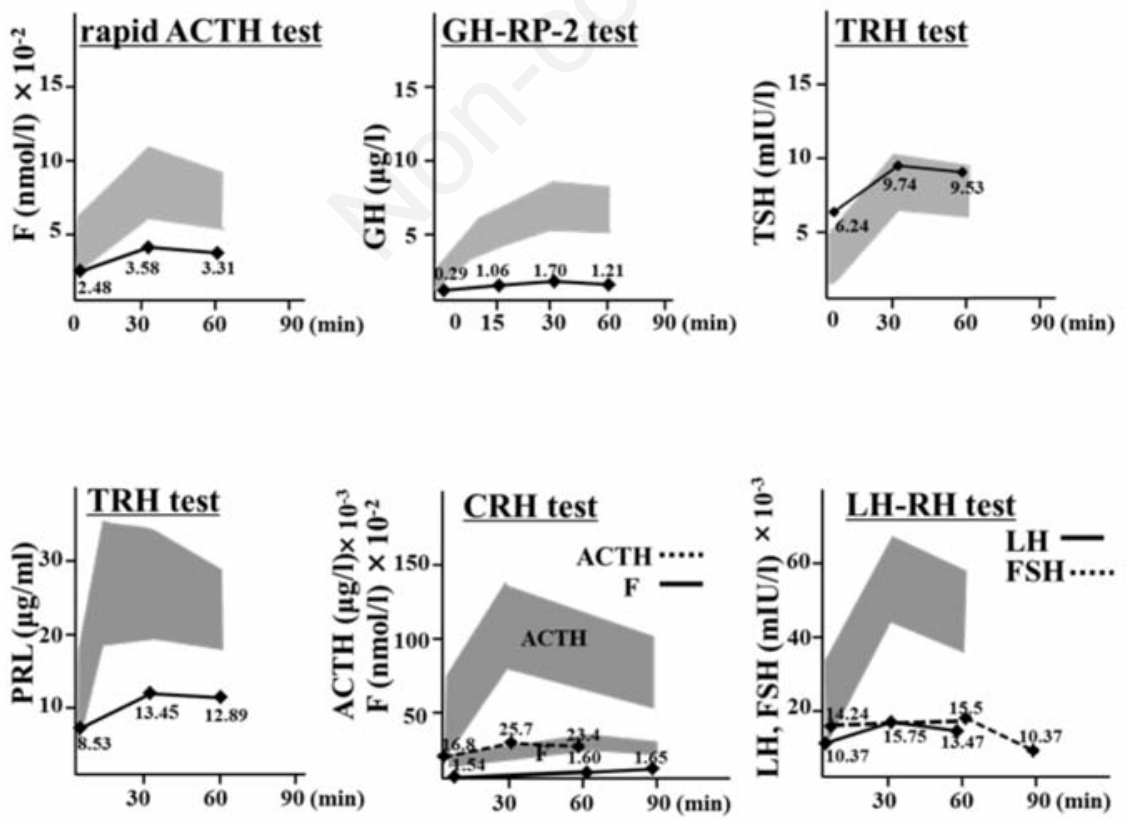

Figure 3. The pituitary hormone stimulating test and rapid ACTH test results. extract from the cytoplasmic fraction of rat pituitary tissue, which was the only method commercially available, even though antigens from the cell membrane would be more related to pathogenesis. In light of the duration of the illness, a patient's APA level may be difficult to detect because the exposure of autoantigen might have ended years or decades earlier, and the antibody titer decreases over time. Regarding the detection of APA in insulindependent diabetes mellitus (which is well known as highly detection of APA disease) patients, Mirakian et al. reported that APA was not detected in most of the patients who had passed several years after an outbreak of the illness, whereas highly detected in the patients who had an outbreak of an illness within 1 year. ${ }^{7}$

However, our patient was revealed to have autoantibodies for thyroid peroxidase, thyroglobulin, and centromere, allowing the diagnosis of chronic thyroiditis and limited scleroderma. Autoimmune thyroiditis in the postpartum period [the so-called post-partum autoimmune thyroiditis (PPAT)] is widely recognized; most patients with PPAT recover transiently, but $20 \%$ of patients shift to chronic thyroiditis. ${ }^{8}$ The frequency of APA detection or lymphocytic hypophysitis in the post-partum period have been reported five times more often among PPAT patients compared to nonPPAT patients, ${ }^{9}$ suggesting that the pathogenesis of PPAT has some common step with that of PAH. The pathogenesis is PPAT is also unknown, but T-lymphocyte activation in the post-partum period that is induced by the cancellation of immunotolerance during the partum period has been proposed. ${ }^{10,11}$ In addition, a graft-versus-host disease (GVHD)-like mechanism induced by immunocompetent cells originating from the fetus after the cancellation of immunotolerance during the partum period has been described.12

In the present case, the patient had autoantibodies for thyroid peroxidase and thyroglobulin, but both titers were at a low level and only the TSH secreting response in the pituitary anterior lobe remained. These two findings strongly support that our patient's thyroiditis had developed a long time ago, at least before her pituitary retraction reached the empty sella.

Regarding our patient's limited scleroderma, she had become aware of Raynaud's phenomenon and sclerodactyly many years ago, suggesting that she had limited scleroderma latently. The interesting hypothesis that systemic scleroderma is a GVHD induced by immunocompetent cells originating from the fetus was proposed, ${ }^{13,14}$ but this concept is controversial. The hypothesis is based on observations of a highly detection of fatal DNA and fatal T-lymphocytes reacting to maternal MHC in the patient's (maternal) blood and skin, and 
a high frequency of fatal HLA-DRB1. In limited scleroderma, the suggestive finding of microchimerism has also been described, although a different pattern of systemic sclerosis (i.e., deletion of fatal DNA in whole blood, or peripheral blood mononuclear cells, and fetomaternal HLA-DRB1compatibility) was observed. 15

\section{Conclusions}

In summary, we treated a patient with latent pregnancy-associated hypopituitarism accompanied by chronic thyroiditis and limited scleroderma. We believe that the hypophysitis, chronic thyroiditis and limited scleroderma began during the patient's second perinatal period, and had been hidden and slowly developed over the following 42 years. An autoimmune mechanism during the perinatal period may possibly be a common step in the pathogenesis of PAH, chronic thyroiditis and limited scleroderma.

\section{References}

1. Sheehan HL. Postpartum necrosis of the anterior pituitary. J Pathol Bacteriol
1937;45:189-214.

2. Goswami R, Kochupillai N, Crock PA, et al. Pituitary autoimmunity in patients with Sheehan's syndrome. J Clin Endocrinol Metab 2002;87:4137-41.

3. De Bellis A, Kelestimur F, Sinisi AA, at al. Anti-hypothalamus and anti-pituitary antibodies may contribute to perpetuate the hypopituitarism in patients with Sheehan's syndrome. Eur J Endocrinol 2008;158:147-52.

4. Cosman F, Post K, Holub D, Wardlaw SL. Lymphocytic hypophysitis. Report of three new cases and review of the literature. Medicine 1989;68:240-56.

5. Patel MC, Guneratne N, Haq N, et al. Peripartum hypopituitarism and lymphocytic hypophysitis. QJ Med 1995;88:571-80.

6. Lupi I, Zhang J, Gutenberg A, et al. From pituitary expansion to empty sella: disease progression in a mouse model of autoimmune hypophysitis. Endocrinology 2011;152:4190-8.

7. Mirakian R, Cudworth AG, Bottazzo GF, et al. Autoimmunity to anterior pituitary cells and the pathogenesis of insulindependent diabetes mellitus. Lancet 1982:755-9.

8. Nicholson WK, Robinson KA, Smallridge $\mathrm{RC}$, et al. Prevalence of postpartum thyroid dysfunction: a quantitative review. Thyroid 2006;16:573-82.

9. Manetti L, Parkes AB, Lupi I, et al. Serum pituitary antibodies in normal pregnancy and in patients with postpartum thyroiditis: a nested case-control study. Eur J Endocrinol 2008;159:805-9.

10. Stagnaro-Green A, Roman SH, Cobin RH, et al. A prospective study of lymphocyteinitiated immunosuppression in normal pregnancy: evidence of a T-cell etiology for postpartum thyroid dysfunction. J Clin Endocrinol Metab 1992;74:645-53.

11. Kuijpens JL, De Hann-Meulman M, Vader HL, et al. Cell-mediated immunity and postpartum thyroid dysfunction: a possibility for the prediction of disease? J Clin Endocrinol Metab 1998;83:1959-66.

12. Ando T, Davies TF. Clinical Review 160: postpartum autoimmune thyroid disease: the potential role of fetal microchimerism. J Clin Endocrinol Metab 2003;88:2965-71.

13. Nelson JL, Furst DE, Maloney S, et al. Microchimerism and HLA-compatible relationships of pregnancy in scleroderma. Lancet 1998;351:559-62.

14. Scaletti C, Vultaggio A, Bonifacio S, et al. Th2-oriented profile of male offspring $\mathrm{T}$ cells present in women with systemic sclerosis and reactive with maternal major histocompatibility complex antigens. Arthritis Rheum 2002;46:445-50.

15. Rak JM, Pagni PP, Tiev K, et al. Male microchimerism and HLA compatibility in French women with sclerodema: a different profile in limited and diffuse subset. Rheumatology (Oxford) 2009;48:363-6. 